\title{
VOLUME COMPARISON FOR HYPERSURFACES IN LORENTZIAN MANIFOLDS AND SINGULARITY THEOREMS
}

\author{
JAN-HENDRIK TREUDE AND JAMES D.E. GRANT
}

\begin{abstract}
We develop area and volume comparison theorems for the evolution of spacelike, acausal, causally complete hypersurfaces in Lorentzian manifolds, where one has a lower bound on the Ricci tensor along timelike curves, and an upper bound on the mean curvature of the hypersurface. Using these results, we give a new proof of Hawking's singularity theorem.
\end{abstract}

\section{INTRODUCTION}

There are many similarities between the ideas inherent in the proof of the singularity theorems in Lorentzian geometry, and those underlying the proofs of certain Riemannian comparison theorems. For example, the interplay between Riccati techniques and index techniques in both fields has been emphasised by Ehrlich [5]. The combination of completeness (which guarantees minimizing geodesics) and curvature conditions (which, via Riccati techniques, imply that geodesics have conjugate points) are the key ingredients in the proof of, for example, Myers's theorem in Riemannian geometry. It is, similarly, the interplay between global hyperbolicity and conjugate points which leads to the singularity theorems in Lorentzian geometry.

We have two objectives in this paper. Firstly, we derive comparison results concerning the area and volume of sets that evolve from a fixed spacelike hypersurface in a Lorentzian manifold. In particular, we prove area and volume monotonicity theorems concerning such quantities, where compared with fixed Lorentzian warped product manifolds. Our techniques are based on ideas from Riemannian geometry (see, e.g., [13). Our second aim is to apply these results to give a new proof of Hawking's singularity theorem [12, pp. 272]. The idea of the proof is that geometrical conditions required for Hawking's result (i.e. that the Ricci tensor be non-negative on timelike vector fields and the initial surface have negative mean curvature) are sufficient to ensure that the volume of the future evolution of the spacelike hypersurface is finite. Combining this property with causal structure arguments then gives the result. Our philosophy here is somewhat similar to that recently employed in the metric measure spaces where Myers's theorem is deduced from a generalised version of the Brunn-Minkowski inequality [19, 14]. In particular, our approach was motivated by the wish to find a method of proof of the singularity theorems that may be generalized to the low-regularity Lorentzian setting.

The plan of the paper is as follows. After recalling necessary background material in Section 2 , we develop the comparison results for Riccati equations that we require in Section 3 In Section 4 we apply these results to derive area and volume monotonicity results for spacelike hypersurfaces in Lorentzian manifolds that satisfy what we call the cosmological comparison condition, $\operatorname{CCC}(\kappa, \beta)$. (See Definition 4.2.) In particular, we introduce comparison geometries in which our area and volume conditions are sharp, and show that geometries satisfying the $\operatorname{CCC}(\kappa, \beta)$ condition satisfy monotonicity properties relative to these model geometries. In Section [5, we show how these geometrical comparison theorems may be applied to give new proofs of the Hawking singularity theorem [12, pp. 272]. Since one of our proofs is based upon geometrical comparison arguments

Date: January 19, 2012. Preprint UWThPh-2012-2.

2010 Mathematics Subject Classification. 53C23, 53C 80.

Key words and phrases. Lorentzian geometry, comparison theorems, singularity theorems.

The authors are grateful to Prof. R. Steinbauer for helpful conversations. JHT would like to thank Stefan Suhr and Olaf Müller for clarifying discussions. The work of JDEG was initially supported by START-project Y237N13 of the Austrian Science Fund A preliminary version of this paper was prepared while JDEG was visiting the Erwin Schrödinger Institute as part of the programme "Dynamics of General Relativity". 
for volumes, areas, etc, it seems plausible that it can be adapted to the low-regularity regime, and, in particular, may be the basis for a proof of the singularity theorems for metrics that are not $C^{2} 1$ After some final remarks, and an outline of some possible directions for further research, in Appendix A, we investigate some conditions under which we may prove a lower bound on the time separation between $\Sigma$ and focal points along normal geodesics. This is essentially an adaption to the Lorentzian regime of the Rauch comparison theorem for submanifolds in Riemannian geometry given by Warner [21]. It leads to comparison theorems where areas and volumes of sets are bounded below in terms of those of corresponding sets in a model geometry.

Notation. Throughout, $(M, \mathbf{g})$ will denote a connected $(n+1)$-dimensional Lorentzian manifold. We will generally use the standard notation of [1, 12, 17]. In particular, we adopt the convention that the metric is of signature $(-,+, \ldots,+)$. We will also denote the product $\mathbf{g}(u, v)$ by $\langle u, v\rangle$. The curvature tensor of the metric is defined with the convention $\mathbf{R}(X, Y) Z=\left(\left[\nabla_{X}, \nabla_{Y}\right]-\nabla_{[X, Y]}\right) Z$, and we denote the Ricci tensor of $\mathbf{g}$ by Ric.

\section{BACKGROUND MATERIAL}

2.1. Causality theory. We first review the concepts that we require from the causal structure theory of Lorentzian manifolds. For extensive, modern reviews of this material, see [4, 15].

Let $(M, \mathbf{g})$ be a connected $(n+1)$-dimensional Lorentzian manifold. Let $p \in M$. A non-zero tangent vector, $v \in T_{p} M$, is said to be timelike, null, or spacelike if $\langle v, v\rangle<0,\langle v, v\rangle=0$, or $\langle v, v\rangle>0$, respectively. A vector that is either timelike or null is called causal. These notions naturally extend to vector fields.

For each $p \in M$, the set of causal vectors in $T_{p} M$ has two connected components, the two causal cones. A time-orientation for $T_{p} M$ is the specification of one of the two causal cones as the future causal cone, and the other one as the past causal cone. A time-orientation for $M$ is a continuous choice of time-orientation in all tangent spaces. A Lorentzian manifold is either timeorientable, or it admits a double-cover that is time-orientable. Therefore, without any important loss of generality, we will assume throughout that our Lorentzian manifolds are time-oriented. We will refer to a Lorentzian manifold with time-orientation as a spacetime.

A piecewise smooth curve 2 in $M$ is called future-directed timelike if its tangent vector is timelike and lies in the future causal cone at all points. Analogously, we define piecewise smooth pastdirected timelike curves and piecewise smooth future- and past-directed causal curves. Given $p, q \in M$, we write $p \ll q$ if there exists a piecewise smooth future-directed timelike curve from $p$ to $q$. Similarly, we write $p<q$ if there exists a piecewise smooth future-directed causal curve from $p$ to $q$. Finally, we write $p \leq q$ if either $p<q$ or $p=q$. Let $A \subseteq M$ be an arbitrary subset of $M$. We define the chronological and causal future of $A$ to be the sets

$$
\begin{aligned}
& I^{+}(A):=\{q \in M \mid \exists p \in A: p \ll q\}, \\
& J^{+}(A):=\{q \in M \mid \exists p \in A: p \leq q\},
\end{aligned}
$$

respectively. Analogously, we define the chronological and causal past $I^{-}(A)$ and $J^{-}(A)$ of $A$.

Finally, we demand that $M$ be globally hyperbolic, i.e. we impose that the following two conditions hold (cf., e.g., [2]):

(1) $M$ is causal, i.e. we have $p \nless p$ for all $p \in M$.

(2) For all $p, q \in M$, the causal diamond $J(p, q):=J^{+}(p) \cap J^{-}(q) \subset M$ is compact.

2.2. Time-separation and maximizing curves. Let $\gamma:[a, b] \rightarrow M$ be a piecewise smooth curve. The Lorentzian arc-length of $\gamma$ is defined to be

$$
L(\gamma)=\sum_{i=1}^{k} \int_{t_{i-1}}^{t_{i}}|\dot{\gamma}(t)| d t
$$

\footnotetext{
${ }^{1}$ This possibility is investigated in 11 .

${ }^{2}$ By a curve, we will mean a continuous map $\gamma: I \rightarrow M$, where $I \subseteq \mathbb{R}$ is an interval.
} 
where $a=t_{0}<\ldots<t_{k}=b$ are the breakpoints of $\gamma$ (i.e. points at which the tangent vector of $\gamma$ is not continuous), and $|\dot{\gamma}(t)|:=\sqrt{|\langle\dot{\gamma}(t), \dot{\gamma}(t)\rangle|}$.

Definition 2.1. The time-separation $\tau: M \times M \rightarrow[0, \infty]$ is defined by

$$
\tau(p, q):=\sup \left\{\begin{array}{l|l}
L(\gamma) & \begin{array}{l}
\gamma \text { is a piecewise smooth future- } \\
\text { directed causal curve from } p \text { to } q
\end{array}
\end{array}\right\}
$$

if $p<q$, and by $\tau(p, q)=0$ if $p \nless q$. If the supremum in (2.1) is attained by a piecewise smooth future-directed causal curve, $\gamma$, from $p$ to $q$, then $\gamma$ is said to be maximizing between $p$ and $q$.

The time-separation may be considered as a Lorentzian analogue of the distance function in Riemannian geometry, although its properties differ in several important respects (see, e.g., 11, Chap. 4]). An important global question concerns the existence of maximizing curves. For globally hyperbolic spacetimes, we have the following well-known result, which illustrates why global hyperbolicity of a Lorentzian manifold may be compared to completeness of a Riemannian manifold from the point of view of arc-length.

Theorem 2.2. Let $(M, \mathbf{g})$ be globally hyperbolic.

(1) For all $p, q \in M$ with $p<q$, there exists a maximizing curve $\gamma:[a, b] \rightarrow M$ from $p$ to $q$. If $p \ll q$, then $\gamma$ may be reparametrized to be a timelike geodesic. Otherwise, $\gamma$ can be reparametrized to be a null geodesic. In both cases, the corresponding geodesic has no conjugate points prior to $q$.

(2) The time-separation of $M$ is finite-valued and continuous.

We shall need a slight variant of the time-separation. Recall that a subset $A \subseteq M$ is said to be acausal if $p \nless q$ for all $p, q \in A$. In particular, if $A$ is acausal then $I^{+}(A) \cap I^{-}(A)=\emptyset$, so we may introduce the signed time-separation to $A, \tau_{A}: M \rightarrow \overline{\mathbb{R}}$, by

$$
\tau_{A}(q):= \begin{cases}\sup _{p \in A} \tau(p, q) & q \in I^{+}(A) \\ -\sup _{p \in A} \tau(q, p) & q \in I^{-}(A) . \\ 0 & \text { else }\end{cases}
$$

In order for these suprema to be attained, global hyperbolicity alone is not sufficient. Rather, one must demand additional compactness properties for $A$. The following concept, introduced by Galloway [10, is well-suited to this purpose.

Definition 2.3. A subset $A \subseteq M$ is future causally complete (FCC), if for each $q \in J^{+}(A)$ the intersection $J^{-}(q) \cap A \subseteq A$ has compact closure in $A$. Similarly, one defines past causal completeness $(\mathrm{PCC})$. A subset that is both FCC and PCC is called causally complete.

Remark 2.4. Clearly every compact set is causally complete. More interestingly, every acausal Cauchy hypersurface is causally complete (cf. [17, Lemma 14.40]). Conversely, one can show that every causally complete, acausal (topological) hypersurface in a globally hyperbolic spacetime is actually a Cauchy hypersurface. This fails if causal completeness is weakened to either only FCC or PCC, and counterexamples are provided by (spacelike) hyperboloids in Minkowski spacetime. Finally, we mention that in globally hyperbolic spacetimes, the notion of causal completeness coincides with the notion of causal compactness as defined in 9, Def. 5.1.1]. Without global hyperbolicity, causal completeness is a weaker condition than causal compactness.

Theorem 2.5. Let $(M, \mathbf{g})$ be globally hyperbolic and $\Sigma \subset M$ a smooth, spacelike, acausal, FCC hypersurface. Then:

(1) For each $q \in J^{+}(\Sigma)$, there exists a point $p \in \Sigma$ with $\tau_{\Sigma}(q)=\tau(p, q)$. Furthermore, the maximizing geodesic from $p$ to $q$ is timelike, normal to $\Sigma$ and has no focal points before $q$.

(2) The signed time-separation of $\Sigma$ is finite-valued and continuous on $J^{+}(\Sigma)$. An analogous result holds for $\Sigma$ being $P C C$ and $q \in J^{-}(\Sigma)$.

Proof. Fix $q \in J^{+}(\Sigma)$. By global hyperbolicity, the function $\tau(\cdot, q): M \rightarrow \mathbb{R}$ is continuous. Therefore, it attains its maximum on the compact subset $3:=\overline{J^{-}(q) \cap \Sigma} \subset \Sigma$ at some point

\footnotetext{
${ }^{3}$ We denote by $\bar{A}$ the closure and by $A^{\circ}$ the interior of a subset $A \subset M$.
} 
$p \in K$. Since $\operatorname{supp} \tau(\cdot, q) \subset J^{-}(q)$, this implies that

$$
\tau(p, q)=\sup _{p^{\prime} \in K} \tau\left(p^{\prime}, q\right)=\sup _{p^{\prime} \in \Sigma} \tau\left(p^{\prime}, q\right)=\tau_{\Sigma}(q) .
$$

The required properties of the maximizing curve are a standard result from the analysis of the index form (see, e.g., [17, Chap. 10]).

2.3. Causal cut locus. Let $M$ be globally hyperbolic and $\Sigma \subset M$ a smooth, spacelike, acausal, FCC hypersurface. Let $N \Sigma \rightarrow \Sigma$ by the normal bundle of $\Sigma \subset M$, and $\exp _{\Sigma}: N \Sigma \rightarrow M$ the normal exponential map. We introduce the future unit-normal bundle

$$
S^{+} N \Sigma:=\{v \in N \Sigma \mid v \text { future-directed, }\langle v, v\rangle=-1\} .
$$

For $v \in S^{+} N \Sigma$, denote by $\gamma_{v}: I_{v} \rightarrow M$ the unique maximal geodesic with $\dot{\gamma}_{v}(0)=v$. It can be shown that (see, e.g., [20, Cor. 3.2.23]), for each $v \in S^{+} N \Sigma, \gamma_{v}$ maximizes the time-separation to $\Sigma$ for small parameter values, in the sense that

$$
\tau_{\Sigma}\left(\gamma_{v}(t)\right)=L\left(\left.\gamma_{v}\right|_{[0, t]}\right) \quad \text { for all sufficiently small } t>0 .
$$

Therefore, for each $v \in S^{+} N \Sigma$, we have

$$
s_{\Sigma}^{+}(v):=\sup \left\{t \in I_{v} \mid \tau_{\Sigma}\left(\gamma_{v}(t)\right)=L\left(\left.\gamma_{v}\right|_{[0, t]}\right)\right\}>0 \text {. }
$$

This defines a function $s_{\Sigma}^{+}: S^{+} N \Sigma \rightarrow(0, \infty]$, called the $\Sigma$-future cut function. If $s_{\Sigma}^{+}(v) \in I_{v}$, then the point $\gamma_{v}\left(s_{\Sigma}^{+}(v)\right)$ is called the $\Sigma$-cut point of $\gamma_{v}$. The collection of such points, i.e. the set

$$
\mathrm{Cut}^{+}(\Sigma):=\left\{\exp _{\Sigma}\left(s_{\Sigma}^{+}(v) v\right) \mid v \in S^{+} N \Sigma \text { and } s_{\Sigma}^{+}(v) \in I_{v}\right\} \subset M,
$$

is called the future cut locus of $\Sigma$.

One can show that a point $q \in M$ lies in $\mathrm{Cut}^{+}(\Sigma)$ if and only if either $q$ is a focal point of $\Sigma$, or if $q$ can be connected to $\Sigma$ by more than one maximizing geodesic. Furthermore, points of the second type are dense in the cut locus 4 Using these properties, one has the following result.

Theorem 2.6. Let $\Sigma \subset M$ be a smooth, spacelike, acausal, FCC hypersurface. Let

$$
\mathcal{J}_{T}^{+}(\Sigma):=\left\{t v \mid v \in S^{+} N \Sigma \text { and } t \in\left[0, s_{\Sigma}^{+}(v)\right)\right\} \subset N \Sigma
$$

and $\mathcal{I}_{T}^{+}(\Sigma)=\mathcal{J}_{T}^{+}(\Sigma)^{\circ}$. Then the following properties hold:

(1) $\mathcal{I}^{+}(\Sigma):=\exp _{\Sigma}\left(\mathcal{I}_{T}^{+}(\Sigma)\right) \subset M$ is open and diffeomorphic to $\mathcal{I}_{T}^{+}(\Sigma)$ via $\exp _{\Sigma}$.

(2) $\mathcal{I}^{+}(\Sigma)=I^{+}(\Sigma) \backslash \mathrm{Cut}^{+}(\Sigma)$.

(3) $\mathrm{Cut}^{+}(\Sigma) \subset M$ has measure zero and is closed.

(4) $\mathcal{I}^{+}(\Sigma)$ is the largest open subset of $I^{+}(\Sigma)$ with the property that each of its points can be connected to $\Sigma$ by a unique maximizing geodesic.

Clearly, if $\Sigma \subset M$ is PCC instead of FCC, then analogous properties hold with future sets replaced with past sets.

2.4. Regularity of the time-separation. One can use Theorem 2.6 to show that on $\mathcal{I}^{+}(\Sigma)$ the signed time-separation $\tau_{\Sigma}$ is actually smooth rather than just continuous.

Proposition 2.7. Let $\Sigma \subset M$ be a smooth, spacelike, acausal, FCC hypersurface. Then the signed time-separation $\tau_{\Sigma}: M \rightarrow \mathbb{R}$ is smooth on $\mathcal{I}^{+}(\Sigma)$ and has the following properties:

(1) For each $q \in \mathcal{I}^{+}(\Sigma)$, we hav日 $\left.\operatorname{grad} \tau_{\Sigma}\right|_{q}=-\dot{\gamma}\left(\tau_{\Sigma}(q)\right)$, where $\gamma:\left[0, \tau_{\Sigma}(q)\right] \rightarrow M$ is the unique maximizing geodesic from $\Sigma$ to $q$, parametrized to unit-speed.

(2) $O n \mathcal{I}^{+}(\Sigma)$, the vector field grad $\tau_{\Sigma}$ is past-directed timelike and has unit-length. Furthermore, grad $\tau_{\Sigma}$ extends to a smooth unit normal for $\Sigma$.

Proof. The first part is shown in a similar way to the analogous statement in Riemannian geometry (cf., e.g., [18, Prop. 4.8]). The second part is an immediate consequence of the first.

\footnotetext{
${ }^{4}$ For proofs of these and the following statements, see [20, Sec. 3.2.5].

${ }^{5}$ Given a $C^{1}$ function, $f$, on $M$ the gradient of $f$ is the vector field defined by the relation $\langle X$, $\operatorname{grad} f\rangle=X(f)$, for all vector fields $X$.
} 
2.5. Level Sets of Distance Functions. Adopting the standard terminology from Riemannian geometry, we will refer to a smooth function $\tau \in C^{\infty}(M)$ that satisfies $\langle\operatorname{grad} \tau, \operatorname{grad} \tau\rangle=-1$ as a timelike distance function. Without loss of generality, we assume that $\operatorname{grad} \tau$ is past-directed (otherwise, consider $-\tau$ ). The principal example that will be of interest to us is the signed timeseparation function of a smooth, spacelike, acausal, FCC hypersurface $\Sigma \subset M$, restricted to $\mathcal{I}^{+}(\Sigma)$ (cf. Proposition 2.7).

Given such a function $\tau$, a short calculation shows that

$$
\nabla_{\operatorname{grad}} \tau \operatorname{grad} \tau=0 .
$$

As a consequence, integral curves of the vector field $\operatorname{grad} \tau$ are (past-directed, timelike, unit-speed) geodesics. In addition, $|\operatorname{grad} \tau|=1$ implies that the map $\tau: M \rightarrow \mathbb{R}$ is a (semi-Riemannian) submersion. Thus the level sets of this map are embedded, spacelike hypersurfaces, which we denote by $\mathcal{S}_{t}:=\tau^{-1}(\{t\}) \subset M$. The restriction of $\operatorname{grad} \tau$ to $\mathcal{S}_{t}$ is a past-directed unit-normal to $\mathcal{S}_{t}$, so the vector field $\mathbf{n}:=-\operatorname{grad} \tau$ yields the corresponding future-directed unit normal.

Consider the subbundle $T \mathcal{S}:=\bigcup_{t \in \operatorname{im}(\tau)} T \mathcal{S}_{t} \subset T M$, and let tan: $T M \rightarrow T \mathcal{S}$ be the corresponding orthogonal projection. For each $t \in \operatorname{im}(\tau)$, let $S_{t} \in \Gamma^{\infty}\left(\operatorname{End}\left(T \mathcal{S}_{t}\right)\right)$ be the shape operator of $\mathcal{S}_{t} \subset M$ with respect to the future-directed unit normal $\mathbf{n}$, which we define with sign convention

$$
S_{t}(w):=\tan \left(\nabla_{w} \mathbf{n}\right)=\nabla_{w} \mathbf{n}+\left\langle\nabla_{w} \mathbf{n}, \mathbf{n}\right\rangle \mathbf{n}=\nabla_{w} \mathbf{n}, \quad w \in T \mathcal{S}_{t} .
$$

Therefore, if we define $S \in \Gamma^{\infty}(\operatorname{End}(T M))$ by $S(X)=\nabla_{X} \mathbf{n}$ for $X \in \Gamma^{\infty}(T M)$, then, for each $t \in \operatorname{im}(\tau)$, the restriction of $S$ to $T \mathcal{S}_{t}$ is the shape operator of $\mathcal{S}_{t} \subset M$ with respect to $\mathbf{n}$. In particular, the corresponding (future) mean curvature $H_{t}:=\operatorname{tr} S_{t} \in C^{\infty}\left(\mathcal{S}_{t}\right)$ is given by

$$
H_{t}(q)=\sum_{i=1}^{n}\left\langle\nabla_{e_{i}} \mathbf{n}, e_{i}\right\rangle=-\sum_{i=1}^{n}\left\langle\nabla_{e_{i}} \operatorname{grad} \tau, e_{i}\right\rangle=-\left.\operatorname{tr} \operatorname{Hess} \tau\right|_{q}=-\square \tau(q),
$$

where $q \in \mathcal{S}_{t}$, and $e_{1}, \ldots, e_{n} \in T_{q} \mathcal{S}_{t}$ is an arbitrarily chosen orthonormal basis. The third equality follows from (2.2) and the fact that $\left.\operatorname{grad} \tau\right|_{q}, e_{1}, \ldots, e_{n}$ is an orthonormal basis of $T_{q} M$.

It will be important to us to know how the family of shape-operators $\left\{S_{t}\right\}_{t}$ changes with respect to the parameter $t$. More precisely, it will be crucial that they obey the following Riccati equation.

Theorem 2.8. Let $S \in \Gamma^{\infty}(\operatorname{End}(T M))$ be given by $S(X)=\nabla_{X} \mathbf{n}$ for $X \in \Gamma^{\infty}(T M)$. Then

$$
\nabla_{\mathbf{n}} S+S^{2}+R_{\mathbf{n}}=0
$$

where $S^{2}=S \circ S$ is to be understood pointwise, and $R_{\mathbf{n}} \in \Gamma^{\infty}(\operatorname{End}(T M))$ denotes the map $X \mapsto \mathbf{R}(X, \mathbf{n}) \mathbf{n}$.

Proof. Let $X \in \Gamma^{\infty}(T M)$, then we have

$$
\begin{aligned}
\left(\nabla_{\mathbf{n}} S\right)(X) & =\nabla_{\mathbf{n}}(S(X))-S\left(\nabla_{\mathbf{n}} X\right) \\
& =\nabla_{\mathbf{n}}\left(\nabla_{X} \mathbf{n}\right)-S\left(\nabla_{\mathbf{n}} X\right) \\
& =\nabla_{X}\left(\nabla_{\mathbf{n}} \mathbf{n}\right)+\mathbf{R}(\mathbf{n}, X) \mathbf{n}+\nabla_{[\mathbf{n}, X]} \mathbf{n}-S\left(\nabla_{\mathbf{n}} X\right) \\
& =-\mathbf{R}(X, \mathbf{n}) \mathbf{n}+S([\mathbf{n}, X])-S\left(\nabla_{\mathbf{n}} X\right) \\
& =-R_{\mathbf{n}}(X)-S^{2}(X) .
\end{aligned}
$$

In the fourth equality, we have used equation (2.2) and, in the last step, the identity $[\mathbf{n}, X]=$ $\nabla_{\mathbf{n}} X-\nabla_{X} \mathbf{n}$.

Properties of solutions of equation (2.4) will be studied in the next section. We conclude this section with two additional results about distance functions that we will require.

Let $\Phi: \mathcal{U} \subset \mathbb{R} \times M \rightarrow M$ be the flow of $\mathbf{n}$, i.e. $\frac{\mathrm{d}}{\mathrm{d} t} \Phi_{t}(p)=\mathbf{n}\left(\Phi_{t}(p)\right)$ for $(t, p) \in \mathcal{U}$. For $p \in M$, we have

$$
\frac{\mathrm{d}}{\mathrm{d} t} \tau\left(\Phi_{t}(p)\right)=\mathrm{d}_{\Phi_{t}(p)} \tau\left(\mathbf{n}_{\Phi_{t}(p)}\right)=\left\langle\left.\mathbf{n}\right|_{\Phi_{t}(p)},\left.\operatorname{grad} \tau\right|_{\Phi_{t}(p)}\right\rangle=1 .
$$

This implies that for $K \subset S_{t}$ and $s \in \mathbb{R}$ such that $\{s\} \times K \subset \mathcal{U}$, we have $\Phi_{s}(K) \subset S_{t+s}$. Using this observation, one can show the following standard result: 
Proposition 2.9 (First Variation of Area). For $t \in \operatorname{im}(\tau)$, let $K \subset \mathcal{S}_{t}$ be compact and assume that the flow, $\Phi$, of $\mathbf{n}$ is defined on $[-\epsilon, \epsilon] \times K$ for some $\epsilon>0$. Set $K_{s}:=\Phi_{s}(K) \subset \mathcal{S}_{t+s}$ for each $s \in[-\epsilon, \epsilon]$. Then

$$
\left.\frac{\mathrm{d}}{\mathrm{d} s}\right|_{s=0} \text { area } K_{s}=\int_{K} \operatorname{tr} S_{t} \mathrm{~d} \mu_{t} .
$$

Here $\mu_{t}$ denotes the Riemannian volume measure of $\left(\mathcal{S}_{t},\left.g\right|_{\mathcal{S}_{t}}\right)$.

Finally, we recall the following version of Fubini's theorem.

Proposition 2.10 (Coarea Formula). For $f \in \mathcal{L}^{1}\left(M, \mathrm{~d} \mu_{g}\right)$, we have $\left.f\right|_{\mathcal{S}_{t}} \in \mathcal{L}^{1}\left(\mathcal{S}_{t}, \mathrm{~d} \mu_{t}\right)$ for almost all $t \in \operatorname{im}(\tau)$ and

$$
\int_{M} f \mathrm{~d} \mu_{g}=\int_{\mathbb{R}}\left(\left.\int_{\mathcal{S}_{t}} f\right|_{\mathcal{S}_{t}} \mathrm{~d} \mu_{t}\right) \mathrm{d} t
$$

\section{RICCATI COMPARISON}

We now abstractly study some properties of solutions of the Riccati equation (2.4). Let $E$ be an $n$-dimensional, real vector space with positive-definite inner product $\langle\cdot, \cdot\rangle$. Denote by $\mathrm{S}(E) \subset$ $\operatorname{End}(E)$ the subspace of linear maps $E \rightarrow E$ that are self-adjoint with respect to $\langle\cdot, \cdot\rangle$. For $A, B \in \mathrm{S}(E)$, we write $A \geq B$ if $A-B \geq 0$ in the sense that $\langle(A-B) v, v\rangle \geq 0$ for all $v \in E$.

We will require the following result from $[8]$ :

Theorem 3.1. Let $R_{1}, R_{2}: \mathbb{R} \rightarrow \mathrm{S}(E)$ be smooth with $R_{1} \geq R_{2}$, in the sense that $R_{1}(t) \geq R_{2}(t)$ for all $t \in \mathbb{R}$. Assume that for $i=1,2$ we have a solution $S_{i}:\left(0, t_{i}\right) \rightarrow \mathrm{S}(E)$ of $S_{i}^{\prime}+S_{i}^{2}+R_{i}=0$, which cannot be extended beyond $t_{i}$. If $U:=S_{2}-S_{1}$ has a continuous extension to $t=0$ with $U(0) \geq 0$, then the following hold.

(1) We have $t_{1} \leq t_{2}$ and $S_{1} \leq S_{2}$ on $\left(0, t_{1}\right)$.

(2) The function $d(t):=\operatorname{dim} \operatorname{ker} U(t)$ is monotonically decreasing on $\left(0, t_{1}\right)$.

(3) If $S_{1}(s)=S_{2}(s)$ for some $s \in\left(0, t_{1}\right)$, then on $(0, s]$ we have $S_{1}=S_{2}$ and $R_{1}=R_{2}$.

Let $R: \mathbb{R} \rightarrow \mathrm{S}(E)$ be smooth, and $S: I^{\prime} \rightarrow \mathrm{S}(E)$ a solution of the Riccati equation

$$
S^{\prime}+S^{2}+R=0
$$

for some interval $I^{\prime} \subseteq \mathbb{R}$. Using Theorem 3.1, we now show that a lower bound on $\operatorname{tr} R$ implies an upper bound on $\operatorname{tr} S$.

Definition 3.2. Let $S: I^{\prime} \rightarrow \operatorname{End}(E)$. We define the expansion $\theta \in C^{\infty}\left(I^{\prime}\right)$, the vorticity $\omega: I^{\prime} \rightarrow$ $\operatorname{End}(E)$, and the shear $\sigma: I^{\prime} \rightarrow \operatorname{End}(E)$ by

$$
\begin{aligned}
\theta(t) & :=\operatorname{tr} S(t), \\
\omega(t) & :=\left(S(t)-S(t)^{\dagger}\right) / 2, \\
\sigma(t) & :=\left(S(t)+S(t)^{\dagger}\right) / 2-\theta(t) / n \cdot \mathrm{id}_{E} .
\end{aligned}
$$

(Recall that $n=\operatorname{dim} E$.)

Taking the trace of the Riccati equation $S^{\prime}+S^{2}+R=0$ and rewriting the quadratic term, one obtains the following result (see, e.g., [12, Chap. 4]).

Lemma 3.3. Let $S: I^{\prime} \rightarrow \operatorname{End}(E)$ be a solution of the Riccati equation (3.1). Then the expansion, vorticity and shear satisfy the scalar Riccati equation

$$
\theta^{\prime}+\frac{\theta^{2}}{n}+\operatorname{tr}\left(\omega^{2}\right)+\operatorname{tr}\left(\sigma^{2}\right)+\operatorname{tr}(R)=0 .
$$

If $S$ is self-adjoint, then (3.3) reduces to the form

$$
\theta^{\prime}+\frac{\theta^{2}}{n}+\operatorname{tr}\left(\sigma^{2}\right)+\operatorname{tr}(R)=0
$$

We now come to the main statement of this section. 
Theorem 3.4. Let $R: \mathbb{R} \rightarrow \mathrm{S}(E)$ be smooth and assume that $\operatorname{tr} R \geq n \cdot \kappa$ for some $\kappa \in \mathbb{R}$ and $n=\operatorname{dim} E$. Furthermore, let $S:(0, b) \rightarrow \mathrm{S}(E)$ be a solution of $S^{\prime}+S^{2}+R=0$, and $s_{\kappa}:\left(0, b_{\kappa}\right) \rightarrow \mathbb{R}$ a solution of $s_{\kappa}^{\prime}+s_{\kappa}^{2}+\kappa=0$ that cannot be extended beyond $b_{\kappa}$. If $\lim _{t \searrow_{0}}\left(s_{\kappa}(t)-\operatorname{tr} S(t) / n\right)$ exists and is nonnegative, then $b \leq b_{\kappa}$ and

$$
\operatorname{tr} S(t) \leq n \cdot s_{\kappa}(t)
$$

for all $t \in(0, b)$. Moreover, if equality holds for some $t_{0} \in(0, b)$, then equality also holds for all $t<t_{0}$. In this case, we also have $S(t)=s_{\kappa}(t) \mathrm{id}_{E}$ and $R(t)=\kappa \cdot \mathrm{id}_{E}$ for all $t \in\left(0, t_{0}\right]$.

Proof. Set $r:=\frac{1}{n}\left(\operatorname{tr}\left(\sigma^{2}\right)+\operatorname{tr}(R)\right)$. By the previous Lemma, $\operatorname{tr} S / n$ obeys the scalar Riccati equation

$$
\left(\frac{\operatorname{tr} S}{n}\right)^{\prime}+\left(\frac{\operatorname{tr} S}{n}\right)^{2}+r=0 .
$$

Furthermore, by assumption we have

$$
r=\frac{\operatorname{tr}\left(\sigma^{2}\right)+\operatorname{tr}(R)}{n} \geq \frac{\operatorname{tr}(R)}{n} \geq \kappa .
$$

Since $s_{\kappa}$ obeys the scalar Riccati equation $s_{\kappa}^{\prime}+s_{\kappa}^{2}+\kappa=0$ and $\lim _{t \searrow 0}\left(s_{\kappa}(t)-\operatorname{tr} S(t) / n\right)$ exists and is nonnegative, we can apply Theorem 3.1. Thus $b \leq b_{\kappa}$ and $\operatorname{tr} S / n \leq s_{\kappa}$, as claimed.

If equality holds for some $t_{0} \in(0, b)$, then by Theorem 3.1 (3) equality also holds for all $t<t_{0}$ and $r(t)=n \cdot \kappa$ for all $t \in\left(0, t_{0}\right]$. From (3.4), it follows that $\operatorname{tr}\left(\sigma(t)^{2}\right)=0$ and $\operatorname{tr} R(t)=n \cdot \kappa$ for all $t \in\left(0, t_{0}\right]$. By the definition, (3.2c), of $\sigma$, the fact that $\operatorname{tr}\left(\sigma(t)^{2}\right)=0$ implies that $\operatorname{tr}\left(S(t)^{2}\right)=$ $n \cdot(\operatorname{tr} S(t))^{2}$. By the Cauchy-Schwarz inequality, this can only hold if $S(t)$ is a multiple of the identity. Thus $S(t)=s_{\kappa}(t)$ id $_{E}$ for all $t \in\left(0, t_{0}\right]$, since $\operatorname{tr} S(t)=n \cdot s_{\kappa}(t)$. Finally, from the Riccati equation for $S$ it follows that $R(t)=\kappa \cdot$ id $_{E}$ for all $t \in\left(0, t_{0}\right]$.

\section{Lorentzian Ricci Curvature Comparison}

In this section, we will establish various comparison theorems for globally hyperbolic Lorentzian manifolds with Ricci curvature bounded from below.

4.1. Notation and Curvature Conditions. In the following, let $M$ be an $(n+1)$-dimensional globally hyperbolic spacetime and $\Sigma \subset M$ a smooth, spacelike, acausal, FCC hypersurface with signed time-separation $\tau_{\Sigma}: M \rightarrow \mathbb{R}$.

Definition 4.1. We define the future spheres and balls of radius $t>0$ around $\Sigma$ to be the sets

$$
S_{\Sigma}^{+}(t):=\tau_{\Sigma}^{-1}(t) \subset I^{+}(\Sigma) \quad \text { and } \quad B_{\Sigma}^{+}(t):=\bigcup_{\tau \in(0, t)} S_{\Sigma}^{+}(\tau) \subset I^{+}(\Sigma) .
$$

For convenience, we set $S_{\Sigma}^{+}(0)=\Sigma$. Furthermore, in order to avoid the cut locus of $\Sigma$, we set $\mathcal{S}_{\Sigma}^{+}(t)=S_{\Sigma}^{+}(t) \cap \mathcal{I}^{+}(\Sigma)$ and $\mathcal{B}_{\Sigma}^{+}(t)=B_{\Sigma}^{+}(t) \cap \mathcal{I}^{+}(\Sigma)$.

By Proposition 2.7, $\tau_{\Sigma}$ is a distance function on $\mathcal{I}^{+}(\Sigma)$, the level sets of which are the restricted future spheres $\mathcal{S}_{\Sigma}^{+}(t)$. From the results of Section 2.5] on $\mathcal{I}^{+}(\Sigma)$, the vector field $\mathbf{n}:=-\operatorname{grad} \tau_{\Sigma}$ is the future-directed timelike unit-normal to the sets $\mathcal{S}_{\Sigma}^{+}(t)$, and the corresponding mean curvature of the hypersurfaces $\mathcal{S}_{\Sigma}^{+}(t)$ is given by

$$
H_{t}(q)=\left.\operatorname{tr} S\right|_{q}=-\square \tau_{\Sigma}(q), \quad q \in \mathcal{S}_{\Sigma}^{+}(t) .
$$

In general, future balls and spheres do not have finite volume and area, respectively 6 Therefore, following [6, 7, we introduce truncated spheres and balls. For $A \subseteq \Sigma$, we set

$$
S_{A}^{+}(t)=\left\{q \in S_{\Sigma}^{+}(t) \mid \exists p \in A: \tau_{\Sigma}(q)=\tau(p, q)\right\},
$$

i.e. $S_{A}^{+}(t) \subseteq S_{\Sigma}^{+}(t)$ consists of those points that can be reached from $A$ by a maximizing geodesic of length $t$. We define $B_{A}^{+}(t)$ similarly, and again we set $\mathcal{S}_{A}^{+}(t)=S_{A}^{+}(t) \cap \mathcal{I}^{+}(\Sigma)$ and $\mathcal{B}_{A}^{+}(t)=$ $B_{A}^{+}(t) \cap \mathcal{I}^{+}(\Sigma)$. If $A$ is compact and $t>0$ is sufficiently small such that $S_{A}^{+}(t)$ does not intersect

\footnotetext{
${ }^{6}$ This is clear in Minkowski spacetime $\mathbb{R}^{n+1}$, choosing $\Sigma=\{0\} \times \mathbb{R}^{n}$.
} 
$\mathrm{Cut}^{+}(\Sigma)$, then $S_{A}^{+}(t) \subset \mathcal{S}_{\Sigma}^{+}(t)$ is also compact, and hence has finite area. Similarly, if $B_{A}^{+}(t)$ does not intersect the causal cut locus of $\Sigma$, it has finite volume by the coarea formula (2.6).

The following curvature conditions will be assumed in all comparison statements.

Definition 4.2. For constants $\kappa, \beta \in \mathbb{R}$, we say the pair $(M, \Sigma)$ satisfies the cosmological comparison condition $\operatorname{CCC}(\kappa, \beta)$ if the following two conditions hold.

(1) $M$ has timelike Ricci curvature bounded from below by $\kappa$, i.e. $\operatorname{Ric}(v, v) \geq n \kappa$ for all $v \in T M$ with $\langle v, v\rangle=-1$.

(2) The mean curvature $H \in C^{\infty}(\Sigma)$ of $\Sigma \subset M$ w.r.t. $\mathbf{n}$ is bounded from above by $\beta$.

Remark 4.3.

(1) $M$ has timelike Ricci curvature bounded from below by $\kappa$ if and only if for any timelike vector $v \in T M$, we have $\operatorname{Ric}(v, v) \geq-n \cdot \kappa\langle v, v\rangle$. The condition $\operatorname{Ric}(v, v) \geq 0$ for all timelike vectors $v \in T M$ is also called the timelike convergence condition or the strong energy condition (cf. [12, pp. 95]).

(2) Recall that $\operatorname{Ric}(v, v)=\langle v, v\rangle \cdot \sum_{i=1}^{n} K\left(v, e_{i}\right)$, where $e_{1}, \ldots, e_{n} \in v^{\perp}$ is an orthonormal basis and $K\left(v, e_{i}\right)$ is the sectional curvature of the plane spanned by $v$ and $e_{i}$. This shows that a lower bound on sectional curvature implies an upper bound on timelike Ricci curvature.

(3) If $(M, \mathbf{g})$ is a Friedmann-Robertson-Walker spacetime, then $\beta$ can be related to the Hubble parameter, i.e. the rate of acceleration of the universe (cf., e.g., [17, pp. 433]). This is the reason for the choice of terminology in Def. 4.2.

4.2. Comparison Geometries. Here we construct certain globally hyperbolic Lorentzian manifolds where the inequalities in the $\operatorname{CCC}(\kappa, \beta)$ condition become equalities. This will lead to a suitable family of comparison spaces.

Our comparison geometries are warped products of the following form. Let $(a, b) \subset \mathbb{R}$ be an interval, $(N, \mathbf{h})$ an $n$-dimensional Riemannian manifold, and $f \in C^{\infty}((a, b))$ a smooth, positive function. We consider the Lorentzian warped product $(M, \mathbf{g})$, where $M=(a, b) \times N$ and $\mathbf{g}$ is given by

$$
\mathbf{g}=-\mathrm{d} t^{2}+f(t)^{2} \mathbf{h} .
$$

We choose the time-orientation such that $\partial_{t}$ is future-directed. We take $(N, \mathbf{h})$ to be complete in order that $(M, \mathbf{g})$ be globally hyperbolic (cf. 11, Sec. 3.6]). In this case, for each $t \in(a, b)$ the hypersurface $N_{t}:=\{t\} \times N \subset M$ is a smooth, spacelike Cauchy hypersurface. In particular, it is acausal and causally complete.

In order to satisfy the lower Ricci curvature bound in Def. 4.2, we will construct comparison spaces that are Einstein, i.e. satisfy $\mathbf{R i c}=-n \kappa \mathbf{g} 7$ A standard curvature calculation implies that this holds if and only if $(N, \mathbf{h})$ is Einstein with $\mathbf{R i c} c_{N}=(n-1) \kappa_{N} \mathbf{h}$ and the warping function satisfies

$$
f^{\prime \prime}=-\kappa \cdot f \quad \text { and } \quad\left(f^{\prime}\right)^{2}+\kappa_{N}=f \cdot f^{\prime \prime} .
$$

For each $\kappa \in \mathbb{R}$ and given initial conditions, there exists a unique maximal solution of the left equation. Separately, for each $\kappa_{N} \in \mathbb{R}$ and given initial conditions, there is a unique maximal solution of the right equation. For certain values of $\kappa, \kappa_{N} \in \mathbb{R}$, these solutions coincide if the initial conditions are chosen appropriately (see Table 1). The two missing cases $\kappa=0, \kappa_{N}>0$ and $\kappa>0, \kappa_{N} \geq 0$ cannot be matched. Note that, rescaling $f$ if necessary, then, without loss of generality, we need only consider the cases $\kappa_{N}=0, \pm 1$.

Regarding the second part of Definition 4.2 we note that for each $t \in(a, b)$ the spacelike hypersurface $N_{t} \subset M$ is totally umbilic and its shape operator with respect to $\partial_{t}$ is given by $S_{t}=\left(f^{\prime}(t) / f(t)\right) \mathrm{id}_{T N_{t}}$. Consequently, the corresponding mean curvature is constant on each $N_{t}$ and given by $H_{t}=\operatorname{tr} S_{t}=n \cdot f^{\prime}(t) / f(t)$. From (4.2), it follows directly that the shape operators satisfy the Riccati equation

$$
S_{t}^{\prime}+S_{t}^{2}+\kappa \cdot \operatorname{id}_{T S_{t}}=0 .
$$

\footnotetext{
${ }^{7}$ The negative sign appears since we want $\operatorname{Ric}(v, v)=n \kappa$ for $\langle v, v\rangle=-1$. (Compare Remark 4.3 (1).)
} 


\begin{tabular}{llll}
$\kappa<0$ & $\kappa_{N}>0$ & $f(t)=\sqrt{\kappa_{N} /|\kappa|} \cosh (\sqrt{|\kappa|} t+b)$ & $H_{t}=n \sqrt{|\kappa|} \tanh (\sqrt{|\kappa|} t+b)$ \\
$\kappa<0$ & $\kappa_{N}=0$ & $f(t)=\mathrm{e}^{ \pm \sqrt{|\kappa|} t}$ & $H_{t}= \pm n \sqrt{|\kappa|}$ \\
$\kappa<0$ & $\kappa_{N}<0$ & $f(t)=\sqrt{\left|\kappa_{N}\right| /|\kappa|} \sinh (\sqrt{|\kappa|} t+b)$ & $H_{t}=n \sqrt{|\kappa|} \operatorname{coth}(\sqrt{|\kappa|} t+b)$ \\
\hline$\kappa=0$ & $\kappa_{N}=0$ & $f(t)=\mathrm{e}^{b}=\operatorname{const}$. & $H_{t}=0$ \\
$\kappa=0$ & $\kappa_{N}<0$ & $f(t)= \pm \sqrt{\left|\kappa_{N}\right|} t+b$ & $H_{t}=n /\left(t \pm b / \sqrt{\left|\kappa_{N}\right|}\right)$ \\
\hline$\kappa>0$ & $\kappa_{N}<0$ & $f(t)=\sqrt{\left|\kappa_{N}\right| / \kappa} \sin (\sqrt{\kappa} t+b)$ & $H_{t}=n \sqrt{\kappa} \cot (\sqrt{\kappa} t+b)$
\end{tabular}

TABLE 1. Warping functions that yield Einstein metrics. $f$ solves the system (4.2). $H_{t}=n f^{\prime}(t) / f(t)$ is the (spatially constant) mean curvature of $N_{t} \subset M$.

We now concretely define our comparison geometries. Let $\kappa, \beta \in \mathbb{R}$ be given. From Table $\mathbb{1}$ one sees that there is a unique way of choosing $\kappa_{N}=0, \pm 1$ and a solution $f_{\kappa, \beta}:\left(a_{\kappa, \beta}, b_{\kappa, \beta}\right) \rightarrow \mathbb{R}$ of (4.2) such that $H_{0}=n \cdot f_{\kappa, \beta}^{\prime}(0) / f_{\kappa, \beta}(0)=\beta$. Here $\left(a_{\kappa, \beta}, b_{\kappa, \beta}\right) \subseteq \mathbb{R}$ is chosen to be the maximal interval containing $t=0$ on which $f_{\kappa, \beta}$ remains strictly positive. Further, we denote by $\left(N_{\kappa, \beta}^{n}, \mathbf{h}_{\kappa, \beta}\right)$ the unique $n$-dimensional, simply-connected space form of constant sectional curvature $\kappa_{N}=0, \pm 1$ as determined by $\kappa, \beta$.

Definition 4.4. Given $\kappa, \beta \in \mathbb{R}$, we denote by $\left(M_{\kappa, \beta}^{n+1}, \mathbf{g}_{\kappa, \beta}\right)$ the warped product

$$
M_{\kappa, \beta}^{n+1}:=\left(a_{\kappa, \beta}, b_{\kappa, \beta}\right) \times N_{\kappa, \beta}^{n}, \quad \mathbf{g}_{\kappa, \beta}:=-\mathrm{d} t^{2}+f_{\kappa, \beta}(t)^{2} \mathbf{h}_{\kappa, \beta},
$$

where $\left(N_{\kappa, \beta}^{n}, \mathbf{h}_{\kappa, \beta}\right)$ and $f_{\kappa, \beta}:\left(a_{\kappa, \beta}, b_{\kappa, \beta}\right) \rightarrow \mathbb{R}$ are as described above. We set $\Sigma_{\kappa, \beta}:=\{0\} \times N_{\kappa, \beta}^{n}$. Then $\Sigma_{\kappa, \beta} \subset M_{\kappa, \beta}^{n+1}$ is a smooth, spacelike, acausal, causally complete hypersurface of constant mean curvature $\beta$. Thus, for the pair $\left(M_{\kappa, \beta}^{n+1}, \Sigma_{\kappa, \beta}\right)$, the $\operatorname{CCC}(\kappa, \beta)$ condition is sharp.

By construction, the signed time-separation $\tau_{\kappa, \beta}: M_{\kappa, \beta}^{n+1} \rightarrow \mathbb{R}$ of $\Sigma_{\kappa, \beta}$ agrees with the function $t:=\operatorname{pr}_{1}: M \rightarrow\left(a_{\kappa, \beta}, b_{\kappa, \beta}\right)$. In particular, the future-directed maximizing geodesics emanating from $\Sigma_{\kappa, \beta}$ are given by the integral curves of $\partial_{t}=-\operatorname{grad} t$. Since integral curves do not cross, it follows that every point in $I^{+}\left(\Sigma_{\kappa, \beta}\right)$ is connected to $\Sigma_{\kappa, \beta}$ by a unique maximizing geodesic. Consequently, we deduce that $\operatorname{Cut}^{+}\left(\Sigma_{\kappa, \beta}\right)=\emptyset$. Further, from $\tau_{\kappa, \beta}=t$, it follows that the future spheres around $\Sigma_{\kappa, \beta}$ are the sets $S_{\kappa, \beta}^{+}(t)=\mathcal{S}_{\kappa, \beta}^{+}(t)=\{t\} \times N_{\kappa, \beta}^{n}$. As noted previously, these hypersurfaces have constant mean curvature $H_{\kappa, \beta}(t)=H_{t}=-\left.\square_{\kappa, \beta} \tau_{\kappa, \beta}\right|_{S_{\kappa, \beta}^{ \pm}(t)}$ w.r.t $\partial_{t}$ (see Table 1 for $H_{t}$ ). It follows from the variation of area formula (2.5) that, for $B \subseteq \Sigma_{\kappa, \beta}$, we have

$$
\operatorname{area}_{\kappa, \beta} S_{B}^{+}(t)=\frac{\operatorname{area}_{\kappa, \beta} B}{f_{\kappa, \beta}(0)^{n}} \cdot f_{\kappa, \beta}(t)^{n} .
$$

The volumes of future balls are obtained by integrating this equation via the coarea formula.

4.3. d'Alembertian and Mean Curvature Comparison. We now prove the first comparison theorem. In the following statements, quantities labelled with indices $\kappa, \beta$ belong to the comparison geometries $\left(M_{\kappa, \beta}^{n+1}, \mathbf{g}_{\kappa, \beta}\right)$ introduced in Sec. 4.2.

Theorem 4.5. Let $\kappa, \beta \in \mathbb{R}$ and assume that $M$ and $\Sigma \subset M$ satisfy the $\operatorname{CCC}(\kappa, \beta)$. Then, for each $q \in \mathcal{I}^{+}(\Sigma)$, we have $\tau_{\Sigma}(q)<b_{\kappa, \beta}$ and

$$
H_{\tau_{\Sigma}(q)}(q)=-\square \tau_{\Sigma}(q) \leq-\square \kappa,\left.\beta \tau_{\kappa, \beta}\right|_{S_{\kappa, \beta}\left(\tau_{\Sigma}(q)\right)}=H_{\kappa, \beta}\left(\tau_{\Sigma}(q)\right) .
$$

Proof. As noted previously, we have $H_{\tau_{\Sigma}(q)}(q)=\left.\operatorname{tr} S\right|_{q}=-\square \tau_{\Sigma}(q)$, and

$$
\nabla_{\mathbf{n}} S+S^{2}+R_{\mathbf{n}}=0
$$

where $R_{\mathbf{n}}=\mathbf{R}(\cdot, \mathbf{n}) \mathbf{n}$.

Fix $q \in \mathcal{I}^{+}(\Sigma)$ and let $\gamma:\left[0, \tau_{\Sigma}(q)\right] \rightarrow M$ be the unique maximizing geodesic from $\Sigma$ to $q$, parametrized to unit-speed. Denote by $\gamma^{\perp} \rightarrow\left[0, \tau_{\Sigma}(q)\right]$ the normal bundle of $\gamma$, i.e. $\gamma_{t}^{\perp}=\dot{\gamma}(t)^{\perp} \subset$ $T_{\gamma(t)} M$ for all $t \in\left[0, \tau_{\Sigma}(q)\right]$. Choose a parallel orthonormal frame $e_{1}, \ldots, e_{n} \in \Gamma^{\infty}\left(\gamma^{\perp}\right)$ and let $e^{1}, \ldots, e^{n} \in \Gamma^{\infty}\left(\left(\gamma^{\perp}\right)^{*}\right)$ be the dual coframe. One can show that $\gamma^{*} S$ and $\gamma^{*} R_{\mathbf{n}}$ take values 
in End $\left(\gamma^{\perp}\right)$. Therefore, we may write $\gamma^{*} S=\mathcal{S}_{j}^{i}\left(e_{i} \otimes e^{j}\right)$ and $\gamma^{*} R_{\mathbf{n}}=\mathcal{R}_{j}^{i}\left(e_{i} \otimes e^{j}\right)$ for smooth functions $\mathcal{S}_{j}^{i}, \mathcal{R}_{j}^{i}:\left[0, \tau_{\Sigma}(q)\right] \rightarrow \mathbb{R}$. Since the frames were chosen orthonormal, and both $R_{\mathfrak{n}}$ and $S$ are (pointwise) self-adjoint w.r.t $\mathbf{g}$, the maps $\mathcal{S}=\left(\mathcal{S}_{j}^{i}\right), \mathcal{R}=\left(\mathcal{R}_{j}^{i}\right):\left[0, \tau_{\Sigma}(q)\right] \rightarrow \operatorname{End}\left(\mathbb{R}^{n}\right)$ are (pointwise) self-adjoint w.r.t. the Euclidean inner product on $\mathbb{R}^{n}$. Further, since $\gamma$ is an integral curve of $\mathbf{n}$ (Proposition 2.7), (4.5) implies that $\mathcal{S}^{\prime}+\mathcal{S}^{2}+\mathcal{R}=0$. By the $\mathrm{CCC}(\kappa, \beta)$-assumption, we have

$$
\operatorname{tr} \mathcal{R}(t)=\operatorname{tr}\{\mathbf{R}(\cdot, \dot{\gamma}(t)) \dot{\gamma}(t)\}=\mathbf{R i c}(\dot{\gamma}(t), \dot{\gamma}(t)) \geq n \cdot \kappa
$$

and

$$
\operatorname{tr} \mathcal{S}(0)=\left.\operatorname{tr} S\right|_{\gamma(0)}=H(\gamma(0)) \leq \beta .
$$

On the other hand, $s_{\kappa, \beta}:=\frac{1}{n} H_{\kappa, \beta}:\left(0, b_{\kappa, \beta}\right) \rightarrow \mathbb{R}$ satisfies the scalar Riccati equation $s_{\kappa, \beta}^{\prime}+s_{\kappa, \beta}^{2}+$ $\kappa=0$ with initial conditions $s_{\kappa, \beta}(0)=\beta$, and cannot be extended beyond $b_{\kappa, \beta}$. Therefore, we can apply the scalar Riccati comparison theorem 3.4 and obtain $\tau_{\Sigma}(q)<b_{\kappa, \beta}$ and $\operatorname{tr} S(\gamma(t)) \leq H_{\kappa, \beta}(t)$ for all $t \in\left(0, \tau_{\Sigma}(q)\right]$. Setting $t=\tau_{\Sigma}(q)$ gives (4.4).

4.4. Area Comparison. We now use the d'Alembertian comparison, together with the variation of area formula (2.5) and the coarea formula (2.6), to obtain comparison statements for areas and volumes of future spheres and balls.

Theorem 4.6. Let $\kappa, \beta \in \mathbb{R}$ and assume that $M$ and $\Sigma \subset M$ satisfy the $\operatorname{CCC}(\kappa, \beta)$. Then, for any $A \subseteq \Sigma$ and $B \subseteq \Sigma_{\kappa, \beta}$, the function

$$
t \mapsto \frac{\operatorname{area} \mathcal{S}_{A}^{+}(t)}{\operatorname{area}_{\kappa, \beta} S_{B}^{+}(t)}, \quad t \in\left[0, b_{\kappa, \beta}\right)
$$

is nonincreasing. Further, for $\tau \searrow 0$, this ratio converges to area $A / \operatorname{area}_{\kappa, \beta} B$, so we also have

$$
\text { area } \mathcal{S}_{A}^{+}(t) \leq \frac{\operatorname{area} A}{\operatorname{area}_{\kappa, \beta} B} \cdot \operatorname{area}_{\kappa, \beta} S_{B}^{+}(t),
$$

for all $t \in\left[0, b_{\kappa, \beta}\right)$.

Remark 4.7. For general $A \subseteq \Sigma$, the sets $\mathcal{S}_{A}^{+}(t) \subseteq \mathcal{S}^{+}(\Sigma, t)$ may not be measurable. In this case, area $\mathcal{S}_{A}^{+}(t)$ should be understood as the inner measure given by $\sup _{K}(\operatorname{area} K)$, where the supremum is taken over all compact sets $K \subset \mathcal{S}_{A}^{+}(t)$. (This will be clear from the proof below.) Since Riemannian measures are Radon measures, this gives the correct result in the measurable case. The same remark applies in the following statements.

Proof. Let $0<t_{1}<t_{2}<b_{\kappa, \beta}$. Choose a sequence of compact sets $K_{i} \subset \mathcal{S}_{A}^{+}\left(t_{2}\right)$ with area $K_{i} \nearrow$ area $\mathcal{S}_{A}^{+}\left(t_{2}\right)$. Each point in $\mathcal{S}_{A}^{+}\left(t_{2}\right)$ can be reached from $\Sigma$ by a unique maximizing, future-directed unit-speed geodesic. Since these geodesics are integral curves of $\mathbf{n}=-\operatorname{grad} \tau_{\Sigma}$ (Proposition 2.7), we have

$$
K_{i}(t):=\Phi_{t-t_{2}}\left(K_{i}\right) \subset \mathcal{S}_{A}^{+}(t),
$$

where $\Phi$ is the flow of $\mathbf{n}$. Further, for each $i \in \mathbb{N}$ and each $t \in\left[0, t_{2}\right], K_{i}(t) \subset \mathcal{S}_{A}^{+}(t)$ is compact and $\Phi$ is defined on $\left(-t, t_{2}-t\right) \times K_{i}(t)$. Therefore, we may use the variation of area formula (2.5), and (4.4), giving

$$
\frac{\mathrm{d}}{\mathrm{d} t} \log \left(\operatorname{area} K_{i}(t)\right)=\frac{1}{\operatorname{area} K_{i}(t)} \int_{K_{i}(t)} H_{t}(q) d \mu_{t}(q) \leq H_{\kappa, \beta}(t) \stackrel{4.3}{=} \frac{\mathrm{d}}{\mathrm{d} t} \log \operatorname{area}_{\kappa, \beta} S_{B}^{+}(t) .
$$

This shows that the function $t \mapsto$ area $K_{i}(t) / \operatorname{area}_{\kappa, \beta} S_{B}^{+}(t)$ is nonincreasing on $\left[0, t_{2}\right]$. Hence

$$
\frac{\operatorname{area} K_{i}\left(t_{2}\right)}{\operatorname{area}_{\kappa, \beta} S_{B}^{+}\left(t_{2}\right)} \leq \frac{\operatorname{area} K_{i}\left(t_{1}\right)}{\operatorname{area}_{\kappa, \beta} S_{B}^{+}\left(t_{1}\right)} \leq \frac{\operatorname{area} \mathcal{S}_{A}^{+}\left(t_{1}\right)}{\operatorname{area}_{\kappa, \beta} S_{B}^{+}\left(t_{1}\right)},
$$

where the final inequality is simply due to the inclusion $K_{i}\left(t_{1}\right) \subseteq \mathcal{S}_{A}^{+}\left(t_{1}\right)$. For $i \rightarrow \infty$, this yields

$$
\frac{\operatorname{area} \mathcal{S}_{A}^{+}\left(t_{2}\right)}{\operatorname{area}_{\kappa, \beta} S_{B}^{+}\left(t_{2}\right)} \leq \frac{\operatorname{area} \mathcal{S}_{A}^{+}\left(t_{1}\right)}{\operatorname{area}_{\kappa, \beta} S_{B}^{+}\left(t_{1}\right)} \text {. }
$$

This shows monotonicity. The second assertion is clear. 
A special case of this result is the following.

Corollary 4.8. Let $\kappa, \beta \in \mathbb{R}$ and assume that $M$ and $\Sigma \subset M$ satisfy the $\mathrm{CCC}(\kappa, \beta)$. Let $A \subseteq \Sigma$ and $B \subseteq \Sigma_{\kappa, \beta}$ with the property that area ${ }_{\kappa, \beta} B=$ area $A$. Then, the function

$$
t \mapsto \frac{\operatorname{area} \mathcal{S}_{A}^{+}(t)}{\operatorname{area}_{\kappa, \beta} S_{B}^{+}(t)}, \quad t \in\left[0, b_{\kappa, \beta}\right)
$$

is nonincreasing, and converges to 1 as $t \searrow 0$. Therefore,

$$
\operatorname{area}_{A}^{+}(t) \leq \operatorname{area}_{\kappa, \beta} S_{B}^{+}(t), \quad t \in\left[0, b_{\kappa, \beta}\right) .
$$

4.5. Volume Comparison. Using the coarea formula (2.6) and the following Lemma, the area comparison theorem immediately yields a volume comparison result.

Lemma 4.9. Let $f, g:[a, b) \rightarrow[0, \infty)$ be locally integrable, nonzero on $(a, b)$, and assume that $f / g$ is non-increasing on $(a, b)$. Then the functions $F, G:(a, b) \rightarrow(0, \infty)$, defined by

$$
F(x)=\int_{a}^{x} f(y) \mathrm{d} y \quad \text { and } \quad G(x)=\int_{a}^{x} g(y) \mathrm{d} y,
$$

are continuous, and $F / G$ is also non-increasing on $(a, b)$.

Proof. Since $f$ and $g$ are locally integrable, $F$ and $G$ are well-defined and continuous. The rest of the proof may be found in [3, pp. 42].

Theorem 4.10. Let $\kappa, \beta \in \mathbb{R}$ and assume that $M$ and $\Sigma \subset M$ satisfy the $\mathrm{CCC}(\kappa, \beta)$. Then, for any $A \subseteq \Sigma$ and $B \subseteq \Sigma_{\kappa, \beta}$, the function

$$
t \mapsto \frac{\operatorname{vol} B_{A}^{+}(t)}{\operatorname{vol}_{\kappa, \beta} B_{B}^{+}(t)}, \quad t \in\left[0, b_{\kappa, \beta}\right)
$$

is nonincreasing. Further, for $t \searrow 0$, this ratio converges to area $A / \operatorname{area}_{\kappa, \beta} B$, so we also have

$$
\operatorname{vol} B_{A}^{+}(t) \leq \frac{\operatorname{area} A}{\operatorname{area}_{\kappa, \beta} B} \cdot \operatorname{vol}_{\kappa, \beta} B_{B}^{+}(t)
$$

for all $t \in\left[0, b_{\kappa, \beta}\right)$.

Proof. By the coarea formula (2.6), for any $t \in\left[0, b_{\kappa, \beta}\right)$, we have

$$
\operatorname{vol} B_{A}^{+}(t)=\int_{0}^{t} \operatorname{area} \mathcal{S}_{A}^{+}(\tau) \mathrm{d} \tau .
$$

Let $0<t_{1}<t_{2}<b_{\kappa, \beta}$ be given. We distinguish two cases. First, assume that vol $B_{A}^{+}\left(t_{2}\right)=\infty$. Then, by (4.6), there exists $\tau_{0} \in(0, t)$ with area $\mathcal{S}_{A}^{+}\left(\tau_{0}\right)=\infty$. By area comparison, we therefore must have area $\mathcal{S}_{A}^{+}(\tau)=\infty$ also for all $\tau<\tau_{0}$. By (4.6) again, it follows that also vol $B_{A}^{+}\left(t_{1}\right)=\infty$, hence the assertion is trivially satisfied.

Now assume that $\operatorname{vol} B_{A}^{+}\left(t_{2}\right)<\infty$. Then by (4.6), the function $\tau \mapsto$ area $\mathcal{S}_{A}^{+}(\tau)$ is locally integrable on $\left[0, t_{2}\right]$. Since also $\tau \mapsto \operatorname{area}_{\kappa, \beta} S_{B}^{+}(t)$ is locally integrable, we may apply Lemma 4.9 together with the area comparison Theorem 4.6. This yields the monotonicity assertion.

Finally, as $t \searrow 0$, we use (4.6) and l'Hôpital's rule to obtain

$$
\lim _{t \searrow 0} \frac{\operatorname{vol} B_{A}^{+}(t)}{\operatorname{vol}_{\kappa, \beta} B_{B}^{+}(t)}=\lim _{t \searrow 0} \frac{\operatorname{area} \mathcal{S}_{A}^{+}(t)}{\operatorname{area}_{\kappa, \beta} S_{B}^{+}(t)}=\frac{\operatorname{area} A}{\operatorname{area}_{\kappa, \beta} B} .
$$

Again, we state a special case of this result.

Corollary 4.11. Let $\kappa, \beta \in \mathbb{R}$ and $(M, \Sigma)$ satisfy the $\operatorname{CCC}(\kappa, \beta)$. Let $A \subseteq \Sigma$ and $B \subseteq \Sigma_{\kappa, \beta}$ be such that $\operatorname{area}_{\kappa, \beta} B=\operatorname{area} A$. Then, the function

$$
t \mapsto \frac{\operatorname{vol} B_{A}^{+}(t)}{\operatorname{vol}_{\kappa, \beta} B_{B}^{+}(t)}, \quad t \in\left[0, b_{\kappa, \beta}\right)
$$


is nonincreasing, and converges to 1 as $t \searrow 0$. Hence,

$$
\operatorname{vol} B_{A}^{+}(t) \leq \operatorname{vol}_{\kappa, \beta} B_{B}^{+}(t), \quad t \in\left[0, b_{\kappa, \beta}\right) .
$$

\section{Application to Singularity Theorems}

We now use the comparison results of the previous section to prove the following singularity theorem due to Hawking [12, pp. 272].

Theorem 5.1. Let $M$ be globally hyperbolic and $\Sigma \subset M$ a smooth, spacelike, acausal, FCC hypersurface 8 Assume that $M$ and $\Sigma$ satisfy the $\operatorname{CCC}(\kappa, \beta)$ with $\kappa=0$ and $\beta<0$. Then no future-directed curve starting in $\Sigma$ can have arc-length greater than $1 /|\beta|$. In particular, $M$ is timelike geodesically incomplete.

Proof via d'Alembertian Comparison. This proof is based on the proof of Myers's theorem in Riemannian geometry given in 22 .

Let $\gamma:[0, b] \rightarrow M$ be a maximizing, timelike, future-directed, unit-speed geodesic emanating perpendicular from $\Sigma$. Then we have $\gamma(t) \in \mathcal{I}^{+}(\Sigma)$ for all $t \in(0, b)$. For $\kappa=0$ and $\beta<0$, the d'Alembertian comparison Theorem 4.5 yields

$$
-\left(\square \tau_{\Sigma}\right)(\gamma(t)) \leq H_{0, \beta}(t)=\frac{1}{t+1 / \beta}=\frac{1}{t-1 /|\beta|},
$$

for all $t \in(0, b)$. Since the right hand side diverges to $-\infty$ for $t \nearrow 1 /|\beta|$, but the left hand side is finite for all $t \in(0, b)$, this implies that $b \leq 1 /|\beta|$. Thus, since every point in $I^{+}(\Sigma)$ can be connected to $\Sigma$ by a maximizing geodesic, we have $\tau_{\Sigma}(q) \leq 1 /|\beta|$ for all $q \in I^{+}(\Sigma)$. From the definition of the time-separation, this gives the required upper bound on arc-length of futuredirected curves starting in $\Sigma$. Timelike geodesic incompleteness follows immediately.

Proof via Area Comparison. We will show that $S_{\Sigma}^{+}(1 /|\beta|) \subset \mathrm{Cut}^{+}(\Sigma)$, which implies that $S_{\Sigma}^{+}(t)=$ $\emptyset$ for all $t>|\beta|$. This again yields $\tau_{\Sigma}(q) \leq 1 /|\beta|$ for all $q \in I^{+}(\Sigma)$, so we can proceed as in the previous proof.

For the sake of contradiction, we assume that there exists $q \in S_{\Sigma}^{+}(1 /|\beta|) \backslash \mathrm{Cut}^{+}(\Sigma)=\mathcal{S}_{\Sigma}^{+}(1 /|\beta|)$. Since the cut locus is closed, there exists a neighborhood $K \subset \mathcal{S}_{\Sigma}^{+}(1 /|\beta|)$ of $q$ with the property that area $K>0$. Set $A:=\Phi_{-1 /|\beta|}(K) \subseteq \Sigma$, where $\Phi$ is the flow of $\mathbf{n}=-\operatorname{grad} \tau_{\Sigma}$, and choose any subset $B \subseteq \Sigma_{0, \beta}$. Then, by the area comparison theorem 4.6 we obtain

$$
\text { area } S_{A}^{+}(t) \leq \frac{\operatorname{area} A}{\operatorname{area}_{0, \beta} B} \cdot \operatorname{area}_{0, \beta} S_{B}^{+}(t) \sim(1-|\beta| t)
$$

for all $t \in(0,1 /|\beta|)$. It follows that area $S_{A}^{+}\left(t_{0}\right)=0$ for some $t_{0} \leq 1 /|\beta|$, and therefore area $K \leq$ area $S_{A}^{+}(1 /|\beta|)=0$. This contradicts the choice of $K$.

\section{FinAL REMARKS}

Our results should be compared with corresponding results in Riemannian geometry. In particular, our proof of the singularity theorem is largely analogous to the proof of Myers's Theorem, which states that a complete Riemannian manifold (of dimension $n$ ) that satisfies the lower Ricci curvature bound Ric $\geq(n-1) \kappa \mathbf{g}$ for some constant $\kappa>0$, is necessarily compact, with diameter less than or equal to $\pi / \sqrt{\kappa} 9$ In a more speculative direction, the area-theoretic approach to the singularity theorems given above may be applicable in more general situations where the Lorentzian metric is of low regularity. For metrics that are not $C^{2}$, one may define a Ricci curvature bound in terms of monotonicity properties of area functionals along geodesics (see, e.g., 16]). Whether one can then develop suitable synthetic-geometrical techniques in Lorentzian geometry to prove singularity theorems for low-regularity metrics is currently under investigation [11.

\footnotetext{
${ }^{8}$ For example, $\Sigma \subset M$ could be a smooth, spacelike Cauchy hypersurface.

${ }^{9}$ In particular, adapting our techniques to develop comparison results for a point instead of a hypersurface, and assuming the stronger curvature bound Ric $\geq \kappa>0$, one can obtain results on existence of conjugate points that are more closely related to Myers's theorem.
} 


\section{Appendix A. Curvature Bounded above}

Let $(M, \mathbf{g})$ be a Lorentzian manifold and $\Sigma$ a smooth spacelike hypersurface in $M$. We finally investigate some conditions under which we may prove a lower bound on the time separation between $\Sigma$ and focal points along normal geodesics.

Proposition A.1. Let $\gamma$ be a normal geodesic to $\Sigma$ parametrized by arc-length. Let $\kappa, \beta \in \mathbb{R}$ be constants such that the curvature operator $R_{\dot{\gamma}}:=\mathbf{R}(\cdot, \dot{\gamma}) \dot{\gamma}$ satisfies $R_{\dot{\gamma}} \leq \kappa \mathrm{id}$, and the shape operator of $\Sigma$ satisfies $S_{\Sigma} \geq \beta$ id. Define the positive constant $t_{0}=t_{0}(\kappa, \beta)$ to be the first positive value of $t$ for which the following equations hold:

$$
\begin{aligned}
\cot (\sqrt{\kappa} t) & =-\frac{\beta}{\sqrt{\kappa}} & & \kappa>0, \\
t & =-\frac{1}{\beta} & & \kappa=0, \\
\operatorname{coth}(\sqrt{|\kappa|} t) & =-\frac{\beta}{\sqrt{|\kappa|}} & & \kappa<0 .
\end{aligned}
$$

(If there are no solutions for positive $t$, set $t_{0}=+\infty$.) Then no point $\gamma(t)$ along the geodesic $\gamma$ is a focal point of $\Sigma$ for $0<t<t_{0}$.

Proof. We proceed in a similar way to the proof of Theorem 4.5 . First, let $s_{\kappa, \beta}:\left[0, b_{\kappa, \beta}\right) \rightarrow \mathbb{R}$ denote the maximal solution of $s_{\kappa, \beta}^{\prime}+s_{\kappa, \beta}^{2}+\kappa=0$ with $s_{\kappa, \beta}(0)=\beta$. We then have $s_{\kappa, \beta}=$ $1 / n \cdot H=f^{\prime} / f$, where the functions $H$ and $f$ may be found in Table 1 (with the constants chosen appropriately). One may check that $t_{0}$ as defined by (A.1) corresponds precisely to the first positive zero of $f$, and hence coincides with $b_{\gamma, \beta}$.

Next, choose an orthonormal frame $e_{1}, \ldots, e_{n} \in \Gamma^{\infty}\left(\gamma^{\perp}\right)$ for the normal bundle of $\gamma$, and let $e^{1}, \ldots, e^{n} \in \Gamma^{\infty}\left(\left(\gamma^{\perp}\right)^{*}\right)$ be the dual coframe. As in the proof of Theorem 4.5, we note that $R_{\dot{\gamma}}$ and the shape operator of the future spheres, $S(X)=-\nabla_{X} \operatorname{grad} \tau_{\Sigma}$, only take values perpendicular to $\gamma$. Therefore, we have $R_{\gamma}=\mathcal{R}_{j}^{i} e_{i} \otimes e^{j}$ and $\gamma^{*} S=\mathcal{S}_{j}^{i} e_{i} \otimes e^{j}$, where the smooth maps $\mathcal{R}=$ $\left(\mathcal{R}_{j}^{i}\right), \mathcal{S}=\left(\mathcal{S}_{j}^{i}\right):[0, T] \rightarrow \operatorname{End}\left(\mathbb{R}^{n}\right)$ are self-adjoint w.r.t the Euclidean inner product and satisfy the Riccati equation $\mathcal{S}^{\prime}+\mathcal{S}^{2}+\mathcal{R}=0$.

Our assumptions are equivalent to $\mathcal{R} \leq \kappa$ id and $\mathcal{S}(0) \geq \beta$ id. Therefore, a direct application of Theorem 3.1 implies that

$$
\mathcal{S}(t) \geq s_{\kappa, \beta}(t) \cdot \mathrm{id} .
$$

Since focal points of $\Sigma$ along $\gamma$ correspond precisely to points at which $\mathcal{S}$ becoming singular in the sense that $\operatorname{tr} \mathcal{S}(t) \searrow-\infty$, it follows from (A.2) and the observation at the beginning of the proof that this situation cannot occur before $t_{0}$ as defined by (A.1). This finishes the proof.

Remarks A.2.

(1) The conditions of Proposition A.1 may alternatively be stated as saying that

$$
\langle\mathbf{R}(X, \dot{\gamma}) \dot{\gamma}, X\rangle \leq \kappa\left(\langle\dot{\gamma}, \dot{\gamma}\rangle\langle X, X\rangle-\langle\dot{\gamma}, X\rangle^{2}\right)
$$

along $\gamma$, for all vector fields $X$ defined along $\gamma$, and that the eigenvalues of the second fundamental form of $\Sigma$ at $p=\gamma(0)$ are bounded below by $\beta$. In this form, Proposition A.1 is essentially an adaption to Lorentzian geometry of the Rauch comparison theorem for submanifolds of Riemannian manifolds 21 .

(2) The estimates in Proposition A.1 are sharp, with equality being achieved for hypersurfaces with all eigenvalues of the shape operator equal to $\beta$ in the two-dimensional, model Lorentzian manifold of constant curvature $\kappa$.

(3) Note that the constant $t_{0}$ is independent of the dimension of the manifold $M$.

Applying Proposition A.1 along all geodesics normal to $\Sigma$, we have the following result.

Theorem A.3. Let $\Sigma \subset M$ be a spacelike hypersurface. Let $\kappa, \beta \in \mathbb{R}$ be given constants. Assume that, for any future-directed geodesic normal to $\Sigma, \gamma:[0, T] \rightarrow M$, normalised such that $\langle\dot{\gamma}, \dot{\gamma}\rangle=$ -1 , the curvature operator $R_{\dot{\gamma}}$ satisfies $R_{\dot{\gamma}} \leq \kappa \mathrm{id}$. Assume further that the shape operator of $\Sigma$ satisfies $S_{\Sigma} \geq \beta$ id. Then no point along $\gamma$ is a focal point of $\Sigma$ if $T<t_{0}$, where $t_{0}$ is as in (A.1). 
We now note that the proof of Proposition A.1 yields the following result.

Proposition A.4. Let $\kappa, \beta \in \mathbb{R}$ and that $(M, \mathbf{g})$ satisfy the conditions of Theorem $A .3$. Then, for $t>0$ sufficiently small such that $S_{\Sigma}^{+}(t) \cap \mathrm{Cut}^{+}(\Sigma)=\emptyset$, the mean curvature $H_{t}$ of $S_{\Sigma}^{+}(t)$ satisfies

$$
H_{t} \geq H_{\kappa, \beta}\left(\tau_{\Sigma}(q)\right),
$$

where $H_{\kappa, \beta}$ are the functions given in Table 1 .

Proof. Take the trace of (A.2).

Following through the proof of Theorem 4.6, we have the following result.

Theorem A.5. Let $\kappa, \beta \in \mathbb{R}$ and assume that $(M, \mathbf{g})$ and $\Sigma \subset M$ satisfy the conditions of Theorem A.3. Then, for any $A \subseteq \Sigma$ and $B \subseteq \Sigma_{\kappa, \beta}$, and $t>0$ sufficiently small that $\mathcal{S}_{A}^{+}(t) \cap$ $\mathrm{Cut}^{+}(\Sigma)=\emptyset$, the map

$$
t \rightarrow \frac{\operatorname{area}_{A}^{+}(t)}{\operatorname{area}_{\kappa, \beta} S_{B}^{+}(t)}
$$

is non-decreasing. Further, for $\tau \searrow 0$, this ratio converges to area $A / \operatorname{area}_{\kappa, \beta} B$, so we have

$$
\text { area } \mathcal{S}_{A}^{+}(t) \geq \frac{\operatorname{area} A}{\operatorname{area}_{\kappa, \beta} B} \cdot \operatorname{area}_{\kappa, \beta} S_{B}^{+}(t),
$$

for all $t \in\left[0, b_{\kappa, \beta}\right)$.

Finally, Lemma 4.9 has no analogue for non-decreasing functions. Therefore, as is standard, there is no relative volume monotonicity theorem in the case of curvature bounded above. Theorem A.5 and the coarea formula, however, yield the following volume comparison result.

Theorem A.6. Let $\kappa, \beta \in \mathbb{R}$ and assume that $(M, \mathbf{g})$ and $\Sigma \subset M$ satisfy the conditions of Theorem A.3. Then, for any $A \subseteq \Sigma$ and $B \subseteq \Sigma_{\kappa, \beta}$, and $t>0$ sufficiently small that $\mathcal{S}_{A}^{+}(t) \cap$ $\mathrm{Cut}^{+}(\Sigma)=\emptyset$, we have

$$
\operatorname{vol} B_{A}^{+}(t) \geq \frac{\operatorname{area} A}{\operatorname{area}_{\kappa, \beta} B} \cdot \operatorname{vol}_{\kappa, \beta} B_{B}^{+}(t)
$$

for all $t \in\left[0, b_{\kappa, \beta}\right)$.

\section{REFERENCES}

[1] J. K. Beem, P. E. Ehrlich, and K. L. Easley, Global Lorentzian geometry, vol. 202 of Monographs and Textbooks in Pure and Applied Mathematics, Marcel Dekker Inc., New York, second ed., 1996.

[2] A. N. Bernal And M. SÁnchez, Globally hyperbolic spacetimes can be defined as 'causal' instead of 'strongly causal', Classical Quantum Gravity, 24 (2007), pp. 745-749.

[3] J. Cheeger, M. Gromov, and M. Taylor, Finite propagation speed, kernel estimates for functions of the Laplace operator, and the geometry of complete Riemannian manifolds, J. Differential Geom., 17 (1982), pp. $15-53$.

[4] P. T. Chruściel, Elements of causality theory. Preprint arXiv:1110.6706

[5] P. E. Ehrlich, Comparison theory in Lorentzian geometry. Lecture notes, Isaac Newton Institute (2005).

[6] P. E. Ehrlich, Y.-T. Jung, And S.-B. Kim, Volume comparison theorems for Lorentzian manifolds, Geom. Dedicata, 73 (1998), pp. 39-56.

[7] P. E. Ehrlich And M. SÁnchez, Some semi-Riemannian volume comparison theorems, Tohoku Math. J. (2), 52 (2000), pp. 331-348.

[8] J.-H. Eschenburg and E. Heintze, Comparison theory for Riccati equations, Manuscripta Math., 68 (1990), pp. 209-214.

[9] F. G. Friedlander, The wave equation on a curved space-time, Cambridge University Press, Cambridge, 1975. Cambridge Monographs on Mathematical Physics, No. 2.

[10] G. J. Galloway, Curvature, causality and completeness in space-times with causally complete spacelike slices, Math. Proc. Cambridge Philos. Soc., 99 (1986), pp. 367-375.

[11] J. D. E. Grant, A synthetic singularity theorem. In preparation, 2012.

[12] S. W. Hawking and G. F. R. Ellis, The large scale structure of space-time, Cambridge University Press, London, 1973. Cambridge Monographs on Mathematical Physics, No. 1.

[13] E. Heintze And H. KARCher, A general comparison theorem with applications to volume estimates for submanifolds, Ann. Sci. École Norm. Sup. (4), 11 (1978), pp. 451-470.

[14] J. Lott and C. Villani, Ricci curvature for metric-measure spaces via optimal transport, Annals of Mathematics, 169 (2009), pp. 903-991. 
[15] E. Minguzzi And M. SÁnchez, The causal hierarchy of spacetimes, in Recent Developments in PseudoRiemannian Geometry, D. Alekseevsky and H. Baum, eds., ESI Lectures in Mathematics and Physics, Zürich, 2008, EMS.

[16] S.-I. OHтA, On the measure contraction property of metric measure spaces, Comment. Math. Helv., 82 (2007), pp. 805-828.

[17] B. O'NeILl, Semi-Riemannian geometry, vol. 103 of Pure and Applied Mathematics, Academic Press Inc., New York, 1983.

[18] T. SAKAI, Riemannian geometry, vol. 149 of Translations of Mathematical Monographs, American Mathematical Society, Providence, RI, 1996.

[19] K.-T. Sturm, On the geometry of metric measure spaces. II, Acta Math., 196 (2006), pp. 133-177.

[20] J.-H. TReude, Ricci curvature comparison in Riemannian and Lorentzian geometry, diploma thesis, Fakultät für Mathematik und Physik, Physikalisches Institut, Albert-Ludwigs-Universität, Freiburg, 2011. Available from http://www.freidok.uni-freiburg.de/volltexte/8405.

[21] F. W. WARNER, Extensions of the Rauch comparison theorem to submanifolds, Trans. Amer. Math. Soc., 122 (1966), pp. 341-356.

[22] S. ZHu, The comparison geometry of Ricci curvature, in Comparison geometry (Berkeley, CA, 1993-94), vol. 30 of Math. Sci. Res. Inst. Publ., Cambridge Univ. Press, Cambridge, 1997, pp. 221-262.

Fakultät für Mathematik, Universität Regensburg, D-93040 Regensburg, Germany

E-mail address: jan-hendrik.treude@mathematik.uni-regensburg.de

$U R L:$ http://homepages .uni-regensburg.de/ trj19484

Gravitationsphysik, Fakultät für Physik, Universität Wien, Boltzmanngasse 5, 1090 Wien, Austria E-mail address: james.grant@univie.ac.at

$U R L:$ http://jdegrant.wordpress.com 